\title{
HPV16/18 E5, a promising candidate for cervical cancer vaccines, affects SCPs, cell proliferation and cell cycle, and forms a potential network with E6 and E7
}

\author{
SHUJIE LIAO, DONGRUI DENG, XIAOJI HU, WEI WANG, LI LI, WEI LI, \\ JIANFENG ZHOU, GANG XU, LI MENG, SHIXUAN WANG and DING MA \\ Cancer Biology Research Center, Tongji Hospital, Tongji Medical College, Huazhong University \\ of Science and Technology, Wuhan, Hubei 430030, P.R. China \\ Received July 28, 2012; Accepted September 21, 2012
}

DOI: $10.3892 /$ ijmm.2012.1168

\begin{abstract}
The spindle checkpoint proteins (SCPs), which sense the existence of misaligned sister chromatids during mitosis and meiosis, are essential for cell proliferation and differentiation. Therefore, the role of SCPs in carcinogenesis is gaining increased attention. In this study, we analysed the expression of Bub1 and Mad2 in clinical samples by immunohistochemistry (IHC) during the development of cervical cancer (CC), and we explored the interaction of Bub1/Mad2 with different proteins through immunoprecipitation (IP). Furthermore, we analysed the characteristics of four different cell models of human papillomavirus (HPV)16/18 E5. We demonstrated that with the progression of CC, the expression of Bub1 and Mad2 was gradually reduced under the influence of HPVE5. Overexpression of HPV16/18 E5 significantly increased cell proliferation, as well as the percentage of cells in the $S$ phase. In addition, the levels of p21, Bub1 and Mad2 were markedly decreased in E5-expressing cells. Therefore, HPV16/18 E5 plays a critical role in carcinogenesis and is a potential therapeutic target in CC treatment.
\end{abstract}

\section{Introduction}

During mitosis, sister chromatids are evenly segregated into daughter cells on the basis of the precise action of spindle checkpoint proteins (SCPs) (1). The SCPs sense the existence

Correspondence to: Professor Ding Ma or Dr Shixuan Wang, Cancer Biology Research Center, Tongji Hospital, Tongji Medical College, Huazhong University of Science and Technology, Wuhan, Hubei 430030, P.R. China

E-mail:dma@tjh.tjmu.edu.cn

E-mail:sxwang@tjh.tjmu.edu.cn

Abbreviations: HPV, human papillomavirus; SCP, spindle checkpoint protein; $\mathrm{CC}$, cervical cancer; $\mathrm{APC} / \mathrm{C}$, anaphase-promoting complex or cyclosome; IHC, immunohistochemistry; CIN, cervical intraepithelial neoplasm; FACS, fluorescence-activated cell sorting; IP, immunoprecipitation; PI, propidium iodide

Key words: human papillomavirus, E5, cervical cancer of misaligned sister chromatids during mitosis and meiosis and use multiple mechanisms to inhibit the ubiquitin ligase activity of the anaphase-promoting complex or cyclosome (APC/C) protein Cdc20 (1). At the same time, the SCPs indirectly preserve chromosome cohesion and delay the onset of sister chromatid separation (1), thus stabilising the cell and delaying the onset of anaphase (2). SCPs are vital in establishing a physical association among sister chromatids in the $S$ phase and maintaining this cohesion until their separation (3). The molecular components of the SCPs include at least two evolutionarily conserved protein families, Mad and Bub (4). Bub1 not only phosphorylates Cdc20 but also interacts with Mad2 by an unknown mechanism (5). Therefore, SCPs are essential for cell proliferation and differentiation. Recently, the role of SCPs in carcinogenesis has gained increased attention.

Alterations in cellular signalling pathways that respond to external stimuli regulating cell mitosis, growth, differentiation and death commonly contribute to cancer. Viruses have developed mechanisms for modulating cellular signalling pathways that reprogram host cells to support their viral life cycles or modulate the host defence responses $(6,7)$. One such case is human papillomavirus (HPV). HPV has been associated with benign and malignant epithelial lesions. In particular, infection with high-risk (HR) HPV (the most epidemic types of which are HPV16/18) is key in cervical cancer (CC) development. In the present study, we investigated whether any changes involving SCPs occur during the development of CC and we explored the possible mechanism by which these proteins change during cancer.

\section{Materials and methods}

Tumour specimens and immunohistochemistry (IHC). Samples of cervical neoplasms at different stages were retrieved from files at the Department of Pathology of Tongji Hospital. The experimental protocols were reviewed and approved by the Institutional Review Board of the Tongji Hospital Ethics Committee. Ninety cervical specimens, which were all HPV16-positive, as determined by HPV DNA gene array (8), were classified as follows: 65 were cervical intraepithelial neoplasm (CIN) biopsy specimens, and included 15 CIN I, 
Table I. HPV16/18 E5 PCR primers for pEGFP-C1 and pcDNA3.1/his.

Gene

pEGFP-C1

pcDNA3.1/his

\section{HPV16 E5 \\ F CCCAAGCTTACTGCATCCACAACATTACTGGC \\ R GGGATCCATTATGTAATTAAAAAGCGTGCA}

\section{HPV18 E5}

\section{F GCGAATTCCATGTTATCACTTATTTTTTTATTTTGC R CCGGATCCCAACCTATACAATTACTGTAAAGACAA}

GGGATCCACCATGTACTGCATCCACAACATTACTGGC AAAGCGGCCGCAATGTAATTAAAAAGCGTGCA

The HindIII, BamHI and EcoRI or BamHI and NotI restriction sites are underlined, and a Kozak motif (11) is incorporated to promote equivalent translational efficiency for constructing and introducing an artificial alanine residue at codon position. F, forward; R, reverse.

23 CIN II and 27 CIN III; 25 were cervical squamous cell carcinoma tissue samples that had been surgically removed. The patients were 23-65 years of age, with a mean age of 43.5 years. Ten samples from healthy women were used as controls.

The sections were deparaffinized in xylene and rehydrated in graded alcohols as described (9). The slides were incubated with a monoclonal antibody against Bub1 (1:50) or Mad2 (1:50) (BD Biosciences, USA).

The slides were analysed by two pathologists who were blinded to the clinical data using light microscopy. Any appreciable brown staining was considered positive and graded as follows: -, no staining; +, barely detectable staining; ++ , easily observed fine granules diffusely present throughout the nucleus or cytoplasm; +++ , staining so strong that nuclear details were obscured. A corresponding H\&E stain was reviewed for determining the diagnosis and mapping the location of the various histological patterns (10).

Immunofluorescence, immunoprecipitation (IP) and western blot analysis. For immunofluorescence, cells were fixed with $4 \%$ paraformaldehyde and incubated with $0.1 \%$ Triton X-100 in PBS, followed by staining the nuclei with propidium iodide (PI).

For the IP, cells were harvested and lysed. The clarified total cell lysates were pre-cleaned with protein $\mathrm{G}$-agarose (Upstate Biotechnology, Inc., USA) for $3 \mathrm{~h}$ at $4^{\circ} \mathrm{C}$, and then incubated with primary antibodies overnight at $4^{\circ} \mathrm{C}$ with gentle rotation. Anti-Bub1, anti-Mad2 (BD Biosciences), anti-GFP or anti-His (Santa Cruz Biotechnology, Inc., USA) antibody was added individually to $1 \mathrm{mg}$ of pre-cleared cell lysate in parallel tests. Immunocomplexes were recovered by incubation with protein $\mathrm{G}$-agarose for $2 \mathrm{~h}$ at $4^{\circ} \mathrm{C}$ on a rotating platform. The antibody and protein $\mathrm{G}$-agarose complexes were separated by SDS-PAGE and analysed by western blot analysis.

For the western blot analysis, proteins were separated by SDS-PAGE and transferred to a polyvinylidene difluoride membrane. The membranes were incubated with primary antibodies [anti-GFP (1:500), anti-His (1:200) (Santa Cruz Biotechnology, Inc.), anti-p21 (1:200), anti-Bub1 (1:200), anti$\operatorname{Mad} 2$ (1:100), or $\beta$-actin antibody (1:500) (BD Biosciences)] overnight at $4^{\circ} \mathrm{C}$. The membranes were then incubated with horseradish peroxidase-conjugated secondary antibody (1: 1,500$)$ for $1 \mathrm{~h}$ at $37^{\circ} \mathrm{C}$ prior to detection by ECL (Pierce, USA).
Construction of recombinant $H P V-16 / 18$ E5 expression plasmids. HPV-16/18 E5 genes were amplified using PCR from a plasmid (pBR322-HPV16/18) that contained the complete genome of HPV16/18 and was kindly provided by Professor Zur Hausen (Heidelberg University, Germany). The PCR primers are shown in Table I (11). The reaction conditions were: 30 denaturation cycles at $94^{\circ} \mathrm{C}$ for $30 \mathrm{sec}$, annealing at $56^{\circ} \mathrm{C}$ for $30 \mathrm{sec}$, and extension at $72^{\circ} \mathrm{C}$ for $45 \mathrm{sec}$. The E5 PCR products were ligated into pEGFP-C1 (Clontech, USA) or pcDNA3.1-V5-His vector (Invitrogen, USA). Positive colonies were screened by PCR and sequenced to confirm the identity of the DNA insertions.

Preparation of stable cell lines expressing HPV E5. The following cell lines were used: $\mathrm{SiHa}$ (a CC cell line that contains an integrated HPV16 genome, ATCC HTB-35 ${ }^{\mathrm{TM}}$ ), HeLa (a CC cell line containing HPV-18 sequences, ATCC CCL-2 ${ }^{\mathrm{TM}}$ ), C33A (a CC cell line negative for HPV DNA and RNA, which was used as control for the potential effects of other HPV components) and the HaCaT cell line (an immortal human keratinocyte cell line from Wuhan University Typical Object Preservation Centre, also used as a control). All cell lines were maintained in DMEM/FCS. Cells were transfected with E5-containing plasmids (HPV16 E5 was transfected into SiHa, C33A, HaCaT cells; HPV18 E5 was transfected into HeLa cells) using Lipofectin ${ }^{\mathrm{TM}}$ (Invitrogen) and grown for 3 weeks in DMEM/FCS containing 600-1,000 mg/l G418 (a cytotoxic dose for non-transfected cells within 1 week). Individual colonies of G418-selected cells were isolated and expanded. The expression of His-E5 or GFP-E5 protein was confirmed using anti-His or anti-GFP antibody in western blot analyses, as a commercial antibody against E5 is not available.

Analysis of gene expression by RT-PCR and real-time RT-PCR. Total-RNA was reverse-transcribed by the RT-PCR System (Life Technologies, USA) (12). The primer sets used are shown in Table II (generated by Takara Biotechnology Co., Ltd., Japan). Real-time RT-PCR was performed on an ABI PRISM 7700 Sequence Detector. Amplified products were detected with SYBR Green PCR Master Mix (PE Biosystems, USA). Each primer set was tested in triplicate for each sample. Realtime RT-PCR was performed for 35 cycles of $15 \mathrm{sec}$ at $95^{\circ} \mathrm{C}$ and $1 \mathrm{~min}$ at $56^{\circ} \mathrm{C}$, preceded by a $2-$ min incubation at $65^{\circ} \mathrm{C}$ 
Table II. Real-time RT-PCR primer sets.

\begin{tabular}{lll}
\hline Gene & \multicolumn{1}{c}{ Forward primer } & \multicolumn{1}{c}{ Reverse primer } \\
\hline Bub1 & GCGACTTTGGATTCTTGTGAGGA & RGGCTGGCTCAGACGAAGTAAGG \\
Mad2 & GTGCAGAAATACGGACTCACCTTG & TTCCAGGACCTCACCACTTTCA \\
P21 & GCTGCGTTCACAGGTGTTTC & CATGGGTTCTGACGGACATC \\
HPV16 E5 & TGACAAATCTTGATACTGCATCCAC & ATAGGCAGACACACAAAAGCACAC \\
HPV18 E5 & CCGCTTTTGCCATCTGTCT & GCAGGGGACGTTATTACCACA \\
GAPDH & ACCCACTCCTCCACCTTTGA & TGACAAAGTGGTCGTTGAGGG
\end{tabular}

Table III. Immunostaining intensity of Bub1 and Mad2 proteins in cervical tissues at various pathological stages with different staining classes.

\begin{tabular}{|c|c|c|c|c|c|c|c|c|c|}
\hline \multirow[b]{2}{*}{ Category } & \multirow[b]{2}{*}{$\mathrm{N}$} & \multicolumn{4}{|c|}{ Bub1 (\%) } & \multicolumn{4}{|c|}{$\operatorname{Mad} 2(\%)$} \\
\hline & & - & + & ++ & +++ & - & + & ++ & +++ \\
\hline $\mathrm{NC}$ & 10 & $0(0.00)$ & $1(10.00)$ & $5(50.00)$ & $4(40.00)$ & $1(10.00)$ & $1(10.00)$ & $5(50.00)$ & $3(30.00)$ \\
\hline CIN & 65 & $21(32.31)$ & $26(40.00)$ & $16(24.62)$ & $2(3.08)$ & $23(35.38)$ & $17(26.15)$ & $21(32.31)$ & $4(6.15)$ \\
\hline CINI & 15 & $1(6.67)$ & $6(40.00)$ & 7 (46.67) & $1(6.67)$ & $0(0.00)$ & $7(46.67)$ & $6(40.00)$ & $2(13.33)$ \\
\hline CINII & 23 & $7(30.43)$ & $10(43.48)$ & $5(21.74)$ & $1(4.35)$ & $9(39.13)$ & $2(8.7)$ & $10(43.48)$ & $2(8.70)$ \\
\hline CINIII & 27 & $13(48.15)$ & $10(37.04)$ & $4(14.81)$ & $0(0.00)$ & $14(51.85)$ & $8(29.63)$ & $5(18.52)$ & $0(0.00)$ \\
\hline $\mathrm{CC}$ & 25 & $16(64.00)$ & $7(28.00)$ & $2(8.00)$ & $0(0.00)$ & $17(68.00)$ & $7(28.00)$ & $1(4.00)$ & $0(0.00)$ \\
\hline SCC & 15 & $10(66.67)$ & $4(26.67)$ & $1(6.67)$ & $0(0.00)$ & $11(77.33)$ & $3(20.00)$ & $1(6.67)$ & $0(0.00)$ \\
\hline $\mathrm{AC}$ & 10 & $6(60.00)$ & $3(30.00)$ & $1(10.00)$ & $0(0.00)$ & $6(60.00)$ & $4(40.00)$ & $0(0.00)$ & $0(0.00)$ \\
\hline
\end{tabular}

NC vs. CIN Bub1 Pearson $\chi^{2}=22.161, \mathrm{P}=0.000$; Mad2 Pearson $\chi^{2}=8.750, \mathrm{P}=0.033$; CIN vs. CC Bub1 Pearson $\chi^{2}=8.382$, P=0.039; Mad2 Pearson $\chi^{2}=11.802, \mathrm{P}=0.008$; NC vs. CC Bub1 Pearson $\chi^{2}=23.713, \mathrm{P}=0.000 ;$ Mad2 Pearson $\chi^{2}=22.001, \mathrm{P}=0.000$; CINI vs. CINII Bub1 Pearson $\chi^{2}=4.342, \mathrm{P}=0.227 ;$ Mad2 Pearson $\chi^{2}=11.608, \mathrm{P}=0.009 ;$ CINII vs. CINIII Bub1 Pearson $\chi^{2}=2.608, \mathrm{P}=0.457 ; \mathrm{Mad} 2 \mathrm{Pearson} \chi^{2}=8.085, \mathrm{P}=0.044$. CINI vs. CINIII Bub1 Pearson $\chi^{2}=10.535, \mathrm{P}=0.015$; Mad2 Pearson $\chi^{2}=13.860, \mathrm{P}=0.003$. NC, normal cervical; CIN, cervical intraepithelial neoplasm; CC, cervical cancer; SCC, squamous cell carcinoma; AC, adenocarcinoma.

and a 10 -min incubation at $95^{\circ} \mathrm{C}$. The instrument software calculated the number of cycles required for the accumulated signal to reach a designated threshold value (cycle threshold, $\mathrm{Ct}$ ) at least 10 standard deviations greater than the baseline. Thus, the $\mathrm{Ct}$ value was proportional to the number of starting copies of the target sequence. The relative quantity of gene expression was calculated using the $\Delta \Delta \mathrm{Ct}$ method, $\Delta \Delta \mathrm{Ct}=[\mathrm{Ct}$ gene of interest (stimulated sample)-Ct GAPDH (stimulated sample)]-[Ct gene of interest (vehicle control)-Ct GAPDH (vehicle control)] (13).

Cell proliferation and cell cycle analyses. Cells were collected and fixed as usual, and then incubated in $\mathrm{Ki}-67$ antibody (mouse) (1:50) (Santa Cruz Biotechnology, Inc.) at $4^{\circ} \mathrm{C}$ overnight. The cells were then incubated in the dark with fluorescein isothiocyanate (FITC)-labeled rabbit anti-mouse immunoglobulin $(1: 1,000)$ (Santa Cruz Biotechnology, Inc.). After an additional wash in PBS, the cells were resuspended and stained with $1 \mathrm{ml}$ of PI/RNase solution (50 g PI and $200 \mathrm{~g}$ RNase/1 in $\mathrm{PBS}$ ) for $15 \mathrm{~min}$ at room temperature before analysing on a FACSCalibur ${ }^{\mathrm{TM}}$ flow cytometer (Becton-Dickinson, USA). The cells were characterised based on green (FITC) and red (PI) fluorescence.
Statistical analysis. Statistical analyses and graphical presentations were performed by SPSS 13.0 and Sigma Plot 10.0, and the results are reported as the means \pm standard error of the mean (SEM). Statistical analysis of significance was based on analysis of variance or $\chi^{2}$ analysis. $\mathrm{P}<0.05$ was considered to indicate statistically significant differences.

\section{Results}

As cervical disease progresses from CIN I to CIN II, CIN III, and finally $C C$, the expression levels of Bubl and Mad2 are gradually suppressed, as shown by IHC. We examined Bubl and Mad2 expression in cervical neoplasm tissue samples by IHC. As cervical disease progressed from CIN I to CIN II, CIN III, and eventually $\mathrm{CC}$, the expression of the two proteins decreased gradually. Indeed, in some cases of CC, Bub1 and Mad2 were undetectable (Table III and Fig. 1). In 10 healthy samples, the levels of Bub1 and Mad2 were significantly higher $(\mathrm{P}<0.01)$ than in the CIN and CC groups. Furthermore, the expression levels in the CIN groups were also significantly different from those in the $\mathrm{CC}$ group $(\mathrm{P}<0.05)$. We concluded that the expression levels of Bub1 and Mad 2 decrease with the severity of cervical disease and the decrease possibly starts from the CIN I stage. 

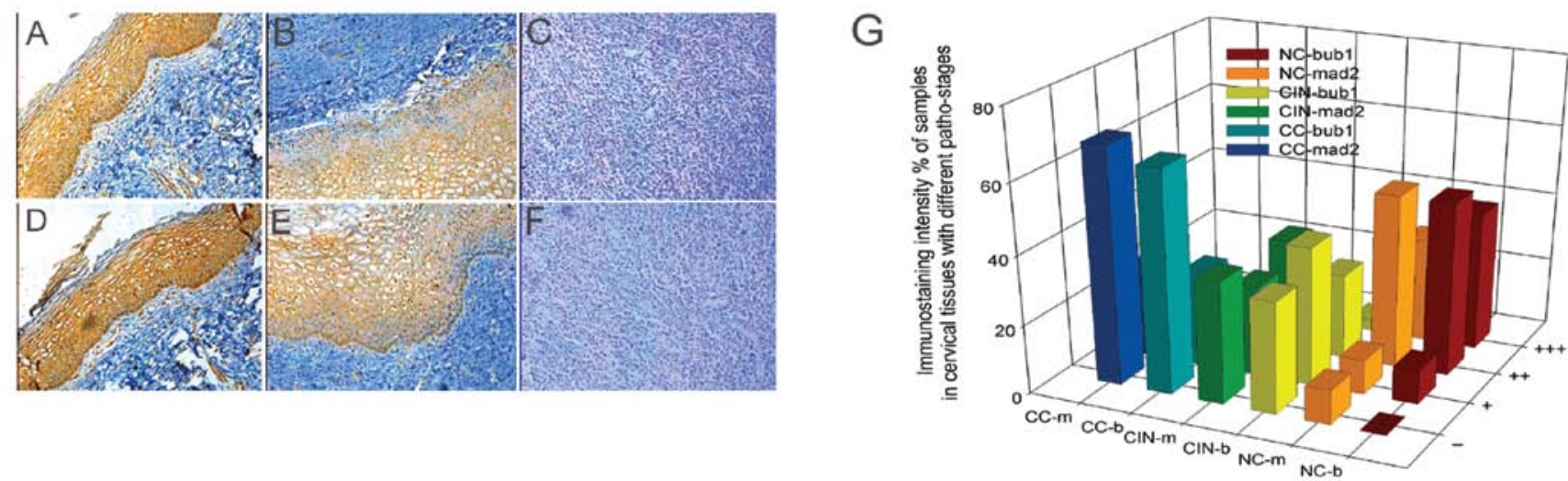

Figure 1. Immunostaining intensity of Bub1 and Mad2 proteins in cervical tissues at various pathological stages with different staining classes by IHC. (A-C) Expression of Bub1 in NC-CIN I, CIN II-III, CC; (D-F) expression of Mad2 in NC-CIN I, CIN II-III, CC; (G) comparison of the expression of Bub1 and Mad2 proteins in cervical tissues with different pathological stages and staining classes. NC, normal cervical; CIN, cervical intraepithelial neoplasm; CC, cervical cancer; b, Bub1; m, Mad2; -, no staining; +, barely detectable staining; ++, easily seen fine granules present diffusely throughout the nucleus or cytoplasm; +++, staining so strong that nuclear detail is obscured.

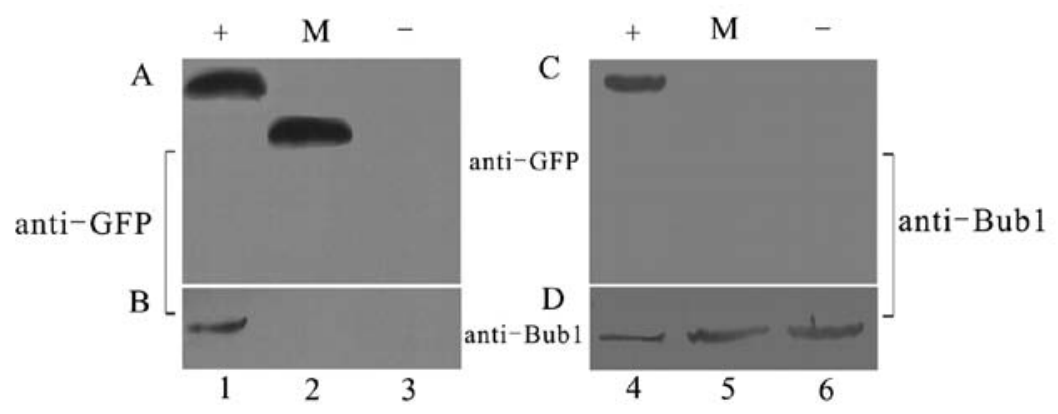

Figure 2. Exploring the relationship between Bub1 and HPV16 E5-GFP by IP and western blot analysis. IP with a GFP antibody, followed by immunoblotting with a Bub1 antibody. (A) GFP+E5 bands in the positive groups (lane 1) and GFP bands in the mock groups (lane 2) are visible, but no bands are visible in the negative groups (lane 3); (B) Bub1 bands in the positive groups were detected (lane 1) but no bands were detected in the mock (lane 2) or negative group (lane 3). IP with a Bub1 antibody, followed by immunoblotting with a GFP antibody. (C) Clear GFP+E5 bands in the positive groups were detected (lane 4) but no bands were detected in the mock (lane 5) or untransfected cells (lane 6); (D) Bub1 bands were detected in all three groups (lanes 4-6) but less was observed in the positive group. +, positive group, re-SiHa cells that were stably transfected with HPV16 E5-pEGFP-C1; -, negative group, SiHa cells; M, mock group, $\mathrm{SiHa}$ cells transfected with the empty plasmids pEGFP-C1.

Interaction between HPV16 E5 and SCPs explored by IP. To explore the mechanism for the downregulation of SCPs in HPV16-related cervical disease, an immunoprecipitation/ immunoblot (IP/IB) experiment was carried out on extracts prepared from primary and transfected cells. We tested several possible target genes, such as HPV16 E6/E7, FLT4, Akt, PI3K and HPV16 E5 (using the HPV16 E5-GFP plasmid from our lab that had been checked by an antibody to GFP) (data not shown). Equivalent amounts of total cell protein from transfected cells were first immunoprecipitated with Bub1 antibody followed by immunoblotting for GFP revealed GFP+E5 bands. Conversely, IP with GFP antibody followed by immunoblotting for Bub1 revealed clear Bub1 bands in the E5-transfected cells, but not in the mock-transfected (empty vector) or untransfected cells (Fig. 2).

These data suggest that Bub1 and HPV16 E5 can interact directly or indirectly in vitro. This result was verified in a parallel experiment using His+E5, which showed exactly the same results (data not shown). Control lanes showed no interaction between GFP and Bub1 (Fig. 2). With the preliminary test, we conducted a thorough experiment.
Expression of Bub1 and Mad2 is significantly suppressed by HPV16/18 E5 further supporting an interaction between HPV16 E5 and Bub1. To analyse the interaction between E5 and Bub1, we tested the most epidemic types of HPV, HPV16/18. We prepared four stable expression HPV16/18 E5-expressing cell lines as described in Materials and methods. As a result, HPV16/18 E5 was expressed in either a His-labelled native form or as a GFP-fusion protein.

We used RT-PCR to determine the expression of HPV16/18 E5 prior to and following transfection. HPV16 E5 was detected in all the transfected cells and at low levels in the control groups (mock-transfected with empty plasmids and untransfected HeLa cells) but was not present in other groups (Fig. 3A). To further substantiate these results and rule out potential effects resulting from the GFP tag, we transfected cells with the His-E5 recombinant plasmids. The protein extracts were blotted with a GFP or His antibody. Bands with compatible molecular weights (GFP+E5 or His+E5) were observed (Fig. 3B and C). When HeLa cells were stably transfected with HPV18 E5, the subcellular localisation of GFP+E5 fluorescence accumulated at the perinuclear region 
A
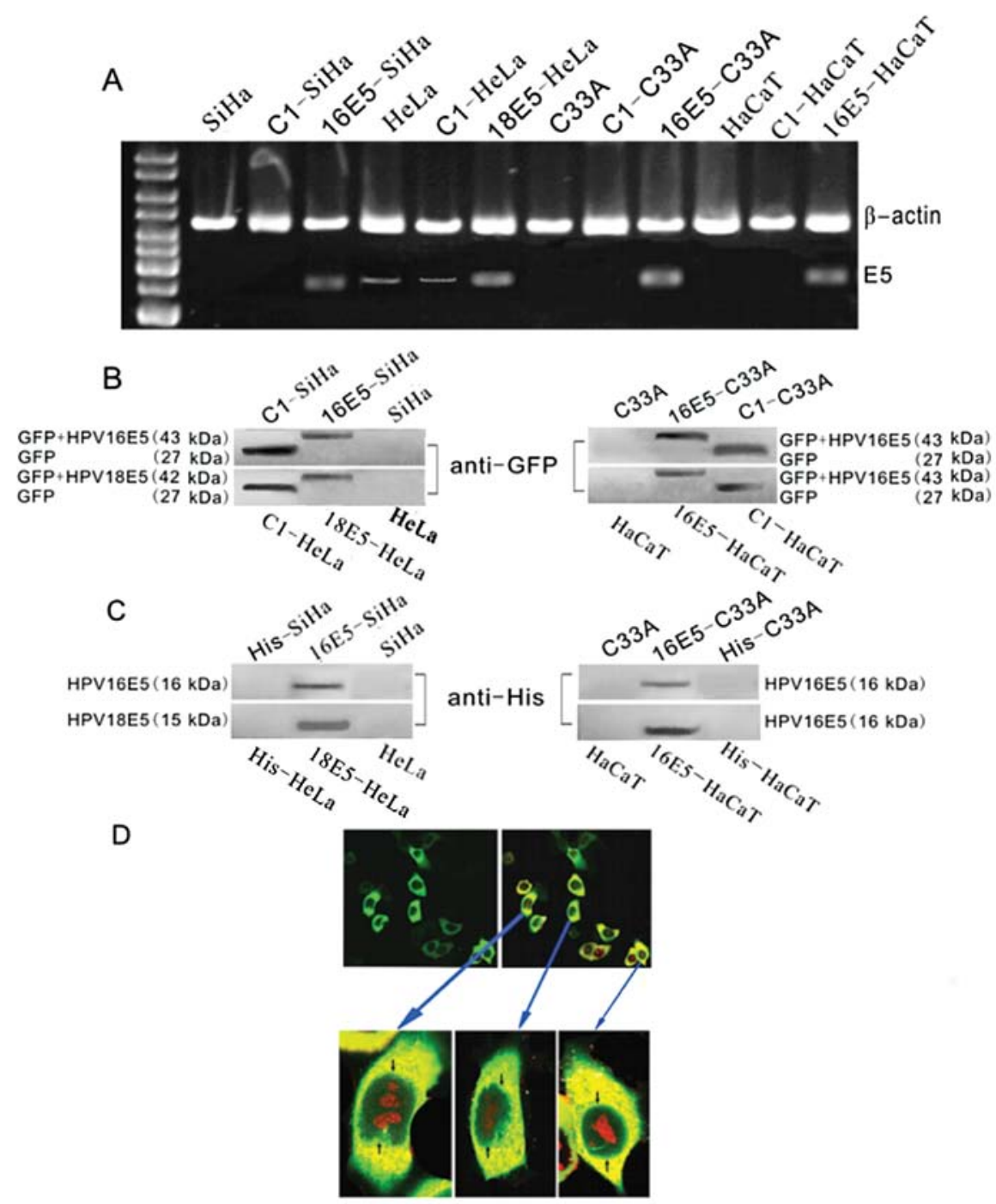

Figure 3. Detection of HPV 16/18 E5 in recombinant plasmids and protein expression verification. (A) RT-PCR for HPV16/18 E5 expression in different cell lines. In the E5-stably transfected cells (16E5-SiHa, 18E5-HeLa, 16E5-C33A and 16E5-HaCaT lanes), E5 was detected. In the empty plasmid pEGFP-C1 transfected groups (C1-SiHa, C1-C33A and C1-HaCaT lanes), E5 was not observed. In the untreated SiHa, C33A and HaCaT cell lanes, no E5 band was found; however, E5 expression in the C1-HeLa group and HeLa cells was scarcely detected. (B and C) Western blot analysis of the GFP and His fusion recombinant proteins of HPV16/18 E5. Using the (B) anti-GFP and (C) anti-His antibodies, respectively, a positive band was observed with a molecular weight corresponding to (B) GFP+HPV16 E5 (43 kDa)/GFP+HPV18 E5 (42 kDa) or (C) His+HPV16 E5 (16 kDa)/His+HPV18 E5 (15 kDa) in the E5-stably transfected cells (16E5-SiHa, 18E5-HeLa, 16E5-C33A, 16E5-HaCaT lanes). In the empty plasmid pEGFP-C1 transfected groups (C1-SiHa, C1-HeLa, C1-C33A and C1-HaCaT lanes), (B) a GFP band was detected (27 kDa), but (C) as the His tag has a low molecular weight, no band was tested in the anti-His group. (B and C) In the untreated $\mathrm{SiHa}, \mathrm{HeLa}, \mathrm{C} 33 \mathrm{~A}$ and $\mathrm{HaCaT}$ cell lanes, no bands were found. (D) Immunofluorescence for detecting the subcellular localization of HPV16 E5, photographed with a confocal fluorescence microscope and nuclei were stained with PI. Most of the fluorescence in GFP+E5 transfectants accumulated at the perinuclear region of the 18E5-HeLa cells that were transfected with pEGFP-C1-HPV18 E5.

of the HeLa cells (Fig. 3D). These results are consistent with those of Krawczyk et al (14).

Additionally, we analysed the relationship between HPV16/18 E5 and Bub1 in all the HPV 16/18 E5 positive groups by western blot analysis and real-time RT-PCR; Bub1 and Mad2 were significantly downregulated (Fig. 4). This result was verified in a parallel experiment using cells that had been stably transfected with His+E5.

HPV16/18 E5 increases cell proliferation and induces cells to leave G1 arrest and enter the $S$ phase. To further investigate the effect of HPV16/18 E5 on the biological characteristics of cervical cells, we performed an additional test. As previously described (15), the expression of the human Ki-67 protein is closely associated with cell proliferation. The $\mathrm{Ki}-67$ protein has been extensively used as a proliferation marker for determining the so-called growth fraction of a given cell population. Therefore, we used FACS to measure the expression of Ki-67. There were significantly higher levels of Ki-67 in the HPV16/18 E5-transfected cells than in the untreated (negative group) and blank vector-transfected cells (mock group) (Fig. 5). Meanwhile, the cycle phase distribution of the cells that were transfected with HPV16/18 E5 was significantly different from the control groups (negative and mock groups) $(\mathrm{P}<0.01)$; a greater proportion was in the $\mathrm{S}$ phase as shown by FACS (Fig. 5). 


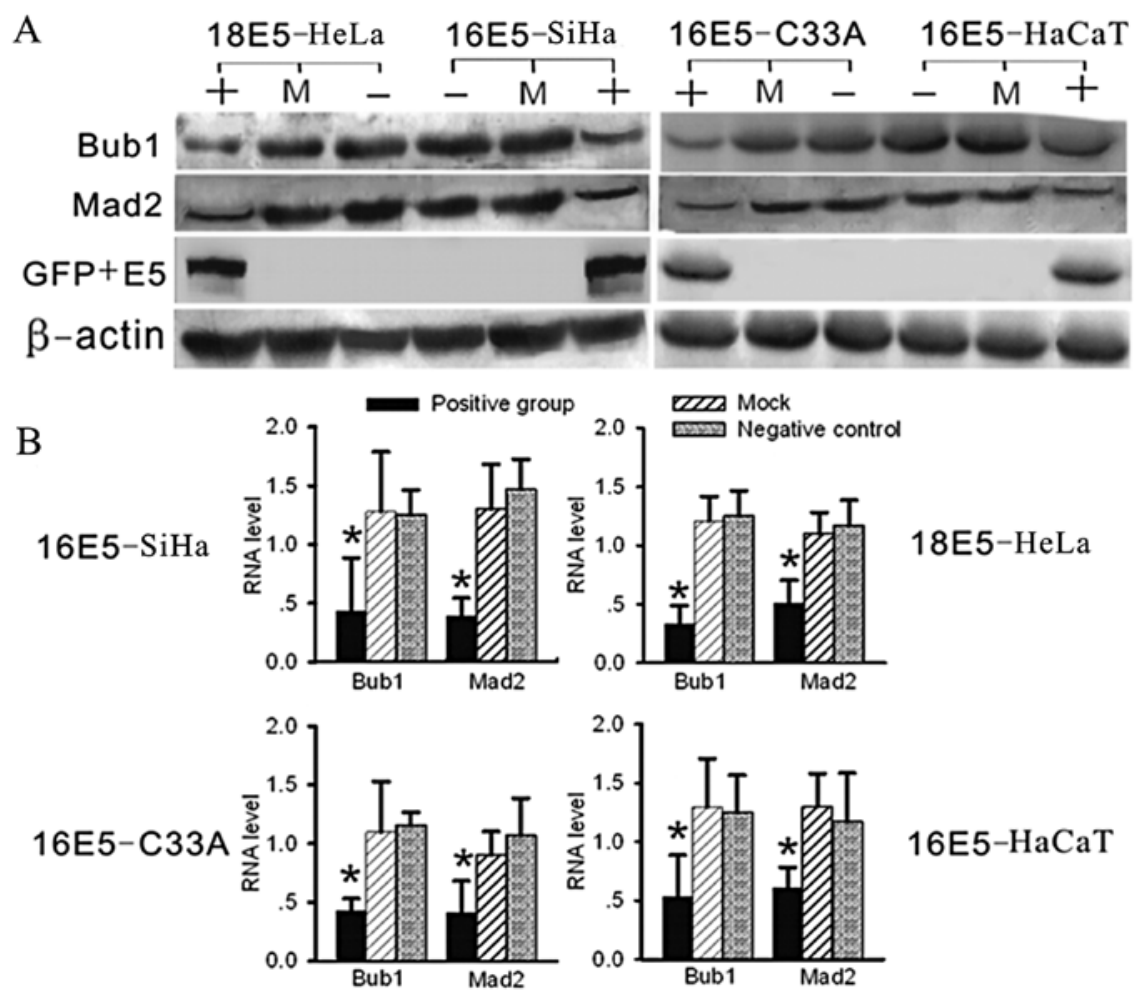

Figure 4. Analysis of RNA and protein levels of Bub1 and Mad2 by real-time RT-PCR and western blot analysis. (A) Using western blot analysis, compared with the negative and mock groups, the expression levels of Bub1 and Mad2 were significantly suppressed in the E5-expressing groups (in two parallel experiments, the pcDNA3.1-V5-His and pcDNA3.1-V5-His-E5 transfected groups showed exactly the same results, data not shown). (B) Compared with the other groups by real-time RT-PCR, the RNA levels of Bub1 and Mad2 were significantly decreased in the E5-expressing groups. (+, positive group, stably transfected with E5-expression plasmids; -, negative group, untreated cells as negative control; M, mock group, transfected with empty vectors, as blank control; * $\mathrm{P}<0.05$ ).
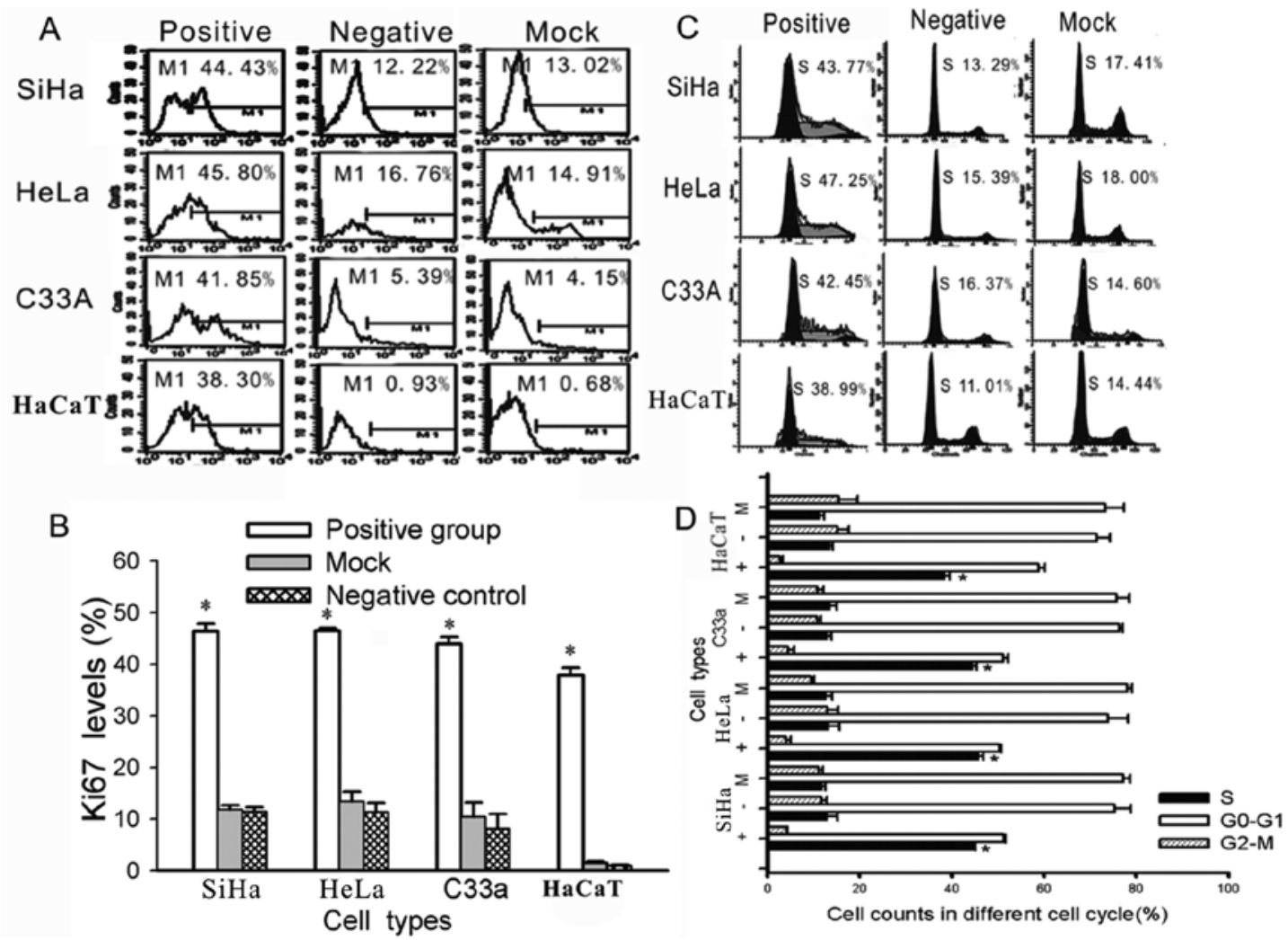

Figure 5. Detection of Ki-67 expression and analysis of cell cycle phase distribution by FACS analysis. (A) The levels of Ki-67 were higher in the positive groups than in the mock and negative groups. (B) Comparison of the level of Ki-67 in different cell lines. (C) The percentage of S phase cells in the positive groups was higher than in the negative and mock groups. (D) Comparison of the percentage of different cell cycle phases in different cells. (+, positive group, stably transfected with HPV16/18 E5; -, negative group, untreated cells as negative control; M, mock group, transfected with empty vectors, as blank control; "P<0.05). 


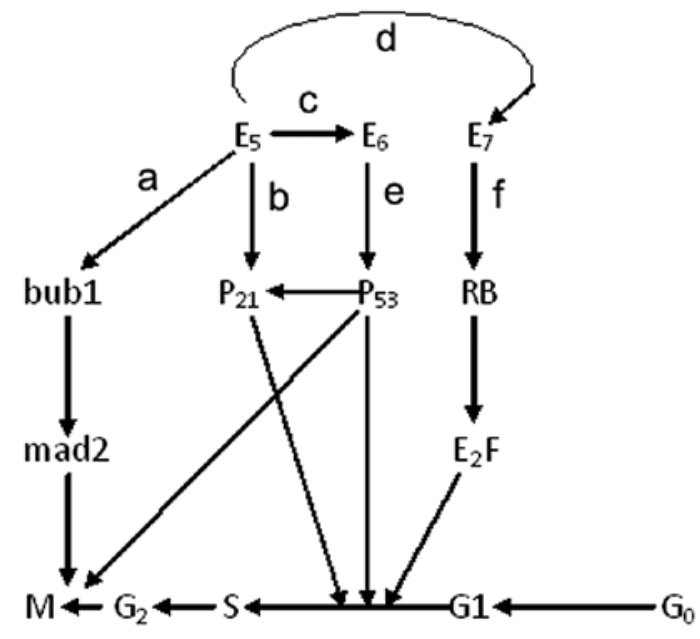

Figure 6. The potential network between E5, E6 and E7 in different cell cycle stages. (a) In our experiment, the down-regulation of the Bub1 and Mad2 may be induced by HPV16 E5 in some ways as proven by IP (Fig. 3) and the expression of Bub1 and Mad2 were decreased significantly in the HPV16/18 E5 stable expression groups on both of the RNA and protein levels (Fig. 5). (b) We also proved that the expression of $\mathrm{p} 21$ (WafI/SdiI/CipI) was reduced in stably expressing E5 cells (Fig. 7). (c) HPV16 E5 enhances the immortalization potential of E6 and E7 (17). Furthermore, E6 targets the tumor suppressor protein $\mathrm{p} 53$, leads to $\mathrm{p} 53$ degradation and contributes to malignant transformation (22), and p53 can upregulate p21 expression (17). (d) The repression of the $\mathrm{p} 21$ gene by the E5 protein may complement the altered $\mathrm{pRb}$ binding activity of mutated E7. (e) E6 proteins increase the turnover of p53, which leads to abrogation of p21-mediated or P21 unrelated G1/S arrest in response to DNA-damaging agents (22). (f) The keratinocytes expressing E7 alone also fail to undergo a G1/S arrest, not directly via p21 suppression but through deregulation of E2F activity (23). Notably, all the details about E5, E6 and E7 can rescue G1 arrest and promote S phase entry in different ways, forming a potential network.

\section{Discussion}

In the present study, we tested 100 specimens from patients with cervical diseases, using IHC, and demonstrated that from CIN I to CIN II, CIN III, and eventually CC, the expression of Bub1 and Mad2 gradually decreased (Table III and Fig. 1). This decrease in the SCPs may influence sister chromatid separation and promote mis-separation producing a heteroploid cell or polyploidy. Given these results, we postulated that an essential mechanism involving these proteins must exist. We examined many possible target genes, such as HPV16 E6/E7, FLT4, Akt and PI3K, and we found that a decrease in the expression of Bub1 and Mad2 may be induced in some manner by HPV16 E5, as shown by IP (Fig. 2). HPV16 E5 is a hydrophobic, 83-amino acid polypeptide that associates with the Golgi apparatus, endoplasmic reticulum, and perinuclear membrane (Fig. 3) $(14,16,17)$. E5 is capable of altering the growth and differentiation of epithelial cells via numerous pathways, including conferring resistance to apoptosis and inducing anchorage-independent growth $(8,18,19)$. Currently, HPV16 E5 is considered an oncogene as it transforms murine fibroblasts and keratinocytes in tissue culture (20) and enhances the immortalisation potential of E6 and E7 (17). However, little is known about the molecular mechanisms by which E5 produces these effects.

To further confirm our results, we prepared four different cell models to test the function of the HR HPV E5 protein (Fig. 3). In our experiment, the expression of Bub1 and Mad2 was significantly decreased in cell lines that stably expressed HPV16/18 E5 at both the RNA and protein levels (Fig. 4). We

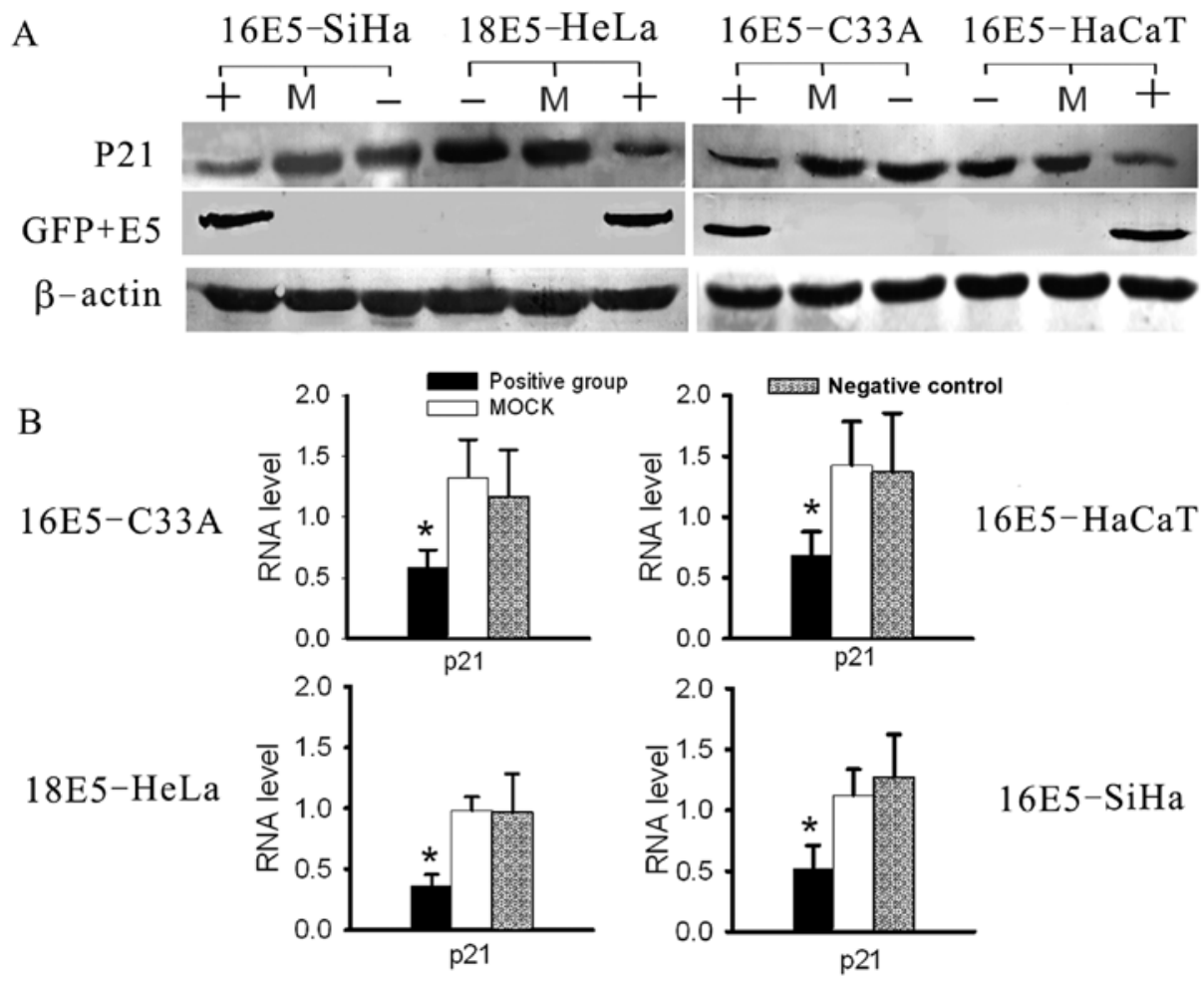

Figure 7. Analysis of the RNA and protein levels of P21 by real-time RT-PCR and western blot analysis. (A) The expression levels of P21 were suppressed in E5-expressing cell lines, compared with the negative and mock groups, as tested by western blot analysis. (B) The RNA levels of P21 were decreased significantly in E5-expressing cells, compared with the other groups, as determined by real-time RT-PCR. (+, positive group, stably transfected with E5-expression vectors; -, negative group, untreated cells as negative control; $\mathrm{M}$, mock group, transfected with empty plasmids, as blank control; ${ }^{*} \mathrm{P}<0.05$ ). 
then investigated the underlying molecular mechanisms and how Bub1, Mad2 and E5 interacted with each other. To do this, we noted that some cytoplasmic organelles (such as the Golgi apparatus and the endoplasmic reticulum) disintegrated to form membrane bubbles during cell prophase when mitotic spindles were emerging $(1,3)$. In addition, we noted that these disintegrated organelles were precisely colocalized with the E5 protein (14). Therefore, we hypothesized that at the beginning of mitosis and at the moment of spindle formation, organelles that were associated with E5 disintegrated and offered a right time and location for E5 and its spindle-related binding partners to associate. This hypothesis would explain the possible interaction between E5 and SCPs. We are currently exploring more direct proof of this interaction.

Next, we thoroughly explored whether there were any changes in cell characteristics as a result of HPV16/18 E5 expression. In this study, we tested the level of Ki-67 by FACS analysis in different groups since the expression of $\mathrm{Ki}-67$ is closely associated with cell proliferation. $\mathrm{Ki}-67$ is absent in resting cells (G0), making it an excellent marker for determining all active phases of the cell cycle $(\mathrm{G} 1, \mathrm{~S}, \mathrm{G} 2$, and mitosis stages). The expression of Ki-67 is also correlated with the clinical course of the disease (15). We showed that HPV16/18 E5-transfected cells had much higher Ki-67 expression and faster proliferation than controls (Fig. 5). Nevertheless, we did not find a significant difference between the HPV-positive and negative groups.

We showed that E5 affected the cell cycle and increased the percentage of $S$ phase cells (Fig. 5), which resulted in increased DNA synthesis. As previously described (21), E6 increases the turnover of $\mathrm{p} 53$, which mediates G1/S arrest in response to DNA-damaging agents through the abrogation of p21 activity (a cyclin-dependent kinase tumour suppressor gene product that causes pocket-protein phosphorylation and releases E2F). Moreover, keratinocytes expressing E7 alone also fail to undergo a $\mathrm{G} 1 / \mathrm{S}$ arrest through the deregulation of E2F activity (22). Each of the E5, E6 and E7 proteins can rescue cells from G1 arrest and promote $S$ phase entry in different ways. They cooperate with and supplement each other, forming a network that regulates the cell cycle (Fig. 6). At the same time, we showed the decreasing expression of $\mathrm{p} 21$ in cells that stably express E5 (Fig. 7). E5 might promote cell proliferation in this way (23). A previous study (24) indicated that intradermal injection of cottontail rabbit papillomavirus DNA, a virus with a natural history of infection similar to that of HPV-16 but with mutations in E7 of critical residues for the binding of $\mathrm{pRb}$, can still induce papilloma formations in rabbits. One possible explanation for these observations is that the suppression of the p21 gene by E5 may facilitate the activation of CDK4-cyclin D complexes, which are known to phosphorylate $\mathrm{pRb}$ and inactivate $\mathrm{Rb}$-checkpoint control (22). Furthermore, E6 leads to p53 degradation and contributes to malignant transformation (21). Since p53 can upregulate p21 expression (17), we also assume that a network including E5, E6, E7, Rb, p53 and p21 exists (Fig. 6).

In conclusion, initial infection with HR HPV causes lowgrade disease and the viral DNA is located in the cell nucleus. E5 is present in the major abundant viral transcripts, as are E6 and E7 (21). During the progression of malignant disease, the HPV DNA integrates into the host cell genome, and E5 is often deleted (25) suggesting that E5 might play an important role at the beginning of HPV infection and in the early stages of HPV-related cervical diseases. The mechanism stimulates cell growth, leads to an increased tendency to enter $\mathrm{S}$ phase, compensates for the function of E6 and E7 and impairs SCPs. These processes would result in aggravation of the malignant transformation potential of HPV-infected cells and acceleration of the carcinogenic process.

\section{Acknowledgements}

This study was supported by grants from the National Science Foundation of China (30901586; 30973205; 30973148); the '973' Program of China (2009CB521808); the Huibei Province Science Foundation (2011CDB191) and the Central University Basic Science Research Foundation (2012QN188).

\section{References}

1. Tang Z, Sun Y, Harley SE, Zou H and Yu H: Human Bub1 protects centromeric sister-chromatid cohesion through Shugoshin during mitosis. Proc Natl Acad Sci USA 101: 18012-18017, 2004.

2. Yu H: Regulation of APC-Cdc20 by the spindle checkpoint. Curr Opin Cell Biol 14: 706-714, 2002.

3. Nasmyth K: Segregating sister genomes: the molecular biology of chromosome separation. Science 297: 559-565, 2002.

4. Cleveland DW, Mao Y and Sullivan KF: Centromeres and kinetochores: from epigenetics to mitotic checkpoint signaling. Cell 112: 407-421, 2003.

5. Tang Z, Shu H, Oncel D, et al: Phosphorylation of Cdc20 by Bub1 provides a catalytic mechanism for APC/C inhibition by the spindle checkpoint. Mol Cell 16: 387-397, 2004.

6. Genther Williams SM, Disbrow GL, Schlegel R, et al: Requirement of epidermal growth factor receptor for hyperplasia induced by E5, a high-risk human papillomavirus oncogene. Cancer Res 65: 6534-6542, 2005.

7. Burgert HG, Ruzsics Z, Obermeier S, et al: A subversion of host defense mechanisms by adenoviruses. Curr Top Microbiol Immunol 269: 273-318, 2002.

8. Vazquez-Ortiz G, Ciudad CJ, Pina P, et al: Gene identification by cDNA arrays in HPV-positive cervical cancer. Arch Med Res 36: 448-458, 2005.

9. Medema RH, Klompmaker R, Smits VA and Rijksen G: P21waf1 can block cells at two points in the cell cycle, but does not interfere with processive DNA replication or stress-activated kinases. Oncogene 16: 431-441, 1998.

10. Freitas S, Moore DH, Michael H and Kelley MR: Studies of apurinic/apyrimidinic endonuclease/ref21 expression in epithelial ovarian cancer: correlations with tumor progression and platinum resistance. Clin Cancer Res 9: 4689-4694, 2003.

11. Kozak M: At least six nucleotides preceding the AUG initiator codon enhance translation in mammalian cells. J Mol Biol 196: 947-950, 1987.

12. Biswas C, Kell B, Mant C, et al: Detection of human papillomavirus type 16 early-gene transcription by reverse transcription-PCR is associated with abnormal cervical cytology. J Clin Microbiol 35: 1560-1564, 1997.

13. Winer J, Jung CK, Shackel I and Williams PM: Development and validation of real-time quantitative reverse transcriptasepolymerase chain reaction for monitoring gene expression in cardiac myocytes in vitro. Anal Biochem 270: 41-49, 1999.

14. Krawczyk E, Suprynowicz FA, Sudarshan SR and Schlegel R: Membrane orientation of the human papillomavirus type 16 E5 oncoprotein. J Virol 84: 1696-1703, 2010.

15. Scholzen T and Gerdes J: The Ki-67 protein: from the known and the unknown. J Cell Physiol 182: 311-322, 2000.

16. Gao $P$ and Zheng J: High-risk HPV E5-induced cell fusion: a critical initiating event in the early stage of HPV-associated cervical cancer. J Virol 7: 238-240, 2010.

17. Boulenouar S, Weyn C, Van Noppen M, et al: Effects of HPV-16 E5, E6 and E7 proteins on survival, adhesion, migration and invasion of trophoblastic cells. Carcinogenesis 31: 473-480, 2010 . 
18. Pedroza-Saavedra A, Lam EW, Esquivel-Guadarrama $\mathrm{F}$ and Gutierrez-Xicotencatl L: The human papillomavirus type 16 E5 oncoprotein synergizes with EGF-receptor signaling to enhance cell cycle progression and the down-regulation of p27(Kip1). Virology 400: 44-52, 2010.

19. Kivi N, Greco D, Auvinen P and Auvinen E: Genes involved in cell adhesion, cell motility and mitogenic signaling are altered due to HPV 16 E5 protein expression. Oncogene 27: 2532-2541, 2008.

20. Nath R, Mant CA, Kell B, et al: Analyses of variant human papillomavirus type-16 E5 proteins for their ability to induce mitogenesis of murine fibroblasts. Cancer Cell Int 6: 19, 2006.

21. Kim MK, Kim HS, Kim SH, et al: Human papillomavirus type 16 E5 oncoprotein as a new target for cervical cancer treatment. Biochem Pharmacol 80: 1930-1935, 2010.
22. Ruesch MN and Laimins LA: Initiation of DNA synthesis by human papillomavirus E7 oncoproteins is resistant to p21-mediated inhibition of cyclin E-cdk2 activity. J Virol 71: 5570-5578, 1997.

23. Tsao YP, Li LY, Tsai TC and Chen SL: Human papillomavirus type 11 and 16 E5 represses p21 (WafI/SdiI/CipI) gene expression in fibroblasts and keratinocytes. J Virol 70: 7535-7539, 1996.

24. Defeo-Jones D, Vuocolo GA, Haskell KM, et al: Papillomavirus E7 protein binding to the retinoblastoma protein is not required for viral induction of warts. J Virol 67: 716-725, 1993.

25. Maufort JP, Shai A, Pitot HC and Lambert PF: A role for HPV16 E5 in cervical carcinogenesis. Cancer Res 70: 2924-2931, 2010. 